\title{
Impactos, vulnerabilidades y desafíos frente al cambio climático
}

\author{
Cristián Henríquez ${ }^{1}$
}

\begin{abstract}
El cambio climático es un fenómeno complejo que se puede clasificar desde un punto de vista científico como emergente y transdisciplinar. En él confluyen aspectos físicos como características del clima: temperatura, precipitación, nubosidad, derretimiento de hielos, salinidad, circulación oceánica, entre otros; como también una serie de aspectos humanos: quema de combustibles fósiles, cambios en los usos/coberturas de suelo, desforestación, entre otros. Todos estos procesos convergen fundamentalmente en la modificación del efecto invernadero producto de la alteración del ciclo de carbono, metano, óxidos de nitrógeno, y otros elementos que alteran la composición de la atmosfera. Las consecuencias globales más significativas son el aumento de la temperatura, cambio en los patrones de precipitaciones, aumento del nivel del mar y también a una serie de eventos desastrosos, como por ejemplo: ciclones, inundaciones, sequías, olas de calor, los cuales impactan directamente a la sociedad. Por cierto también pueden haber impactos positivos, pero la atención de la política pública está puesta en prevenir y adaptarse a los efectos más negativos del cambio climático.
\end{abstract}

En este contexto, este número semitemático presenta algunos resultados y reflexiones de distintos estudios que se están desarroIlando y que pueden ser de utilidad para iniciar una discusión sobre los desafíos que el cambio climático supone a nivel regional y local. Estas contribuciones, que surgen a partir de un Seminario realizado en enero de

\footnotetext{
1 Instituto de Geografía, Pontificia Universidad Católica de Chile (Chile). E-mail: cghenriq@uc.cl
}

2013 bajo el contexto del Proyecto Fondecyt 1100657, pretenden ilustrar distintas dimensiones y escalas del problema y dar algunas luces de cómo enfrentarlo desde una perspectiva geográfica.

El primer artículo, de Ranyére Silva Nóbrega y Gabriela Ayane Chagas Felipe Santiago, analiza las tendencias de temperatura de superficie del mar (TSM), mediante un índice de anomalías de lluvias, y lo relaciona con la variabilidad temporal de precipitaciones en el noreste de Brasil. Los análisis estadísticos de la TSM muestran tendencias de calentamiento, mientras que los análisis de datos aislados, demuestran que cuando hay ocurrencia de anomalías positivas sobre el Pacífico este (El Niño) y anomalías negativas sobre el Atlántico sur, las sequías son más severas. Los impactos socioeconómicos estimados para la región del sertão serían fundamentalmente para el sector agrícola y las familias rurales que dependen de estas actividades, en particular las familias más pobres. En consecuencia también se esperarían aumento de los flujos migratorios campo-ciudad, especialmente en la región metropolitana de Recife y en otras ciudades del estado de Pernambuco.

Cristián Henríquez, Nicolle Aspee y Jorge Quense analizan de manera retrospectiva los eventos climáticos extremos asociados a declaratorias de zonas de catástrofes en Chile (1984-2013) por temporales de lluvia, sequías, heladas, nevazones, inundaciones y movimientos en masa desencadenados por temperaturas y precipitaciones extremas. Además proponen un índice de riesgo hidrometeorológico, en base a información de exposición, vulnerabilidad y resiliencia en base a fuentes oficiales. De acuerdo a los 
resultados obtenidos las comunas del país que presentan mayor riesgo climático son las ubicadas en el litoral de la zona central. Se concluye sobre la necesidad de usar un enfoque adaptativo y no reactivo en el manejo del riesgo especialmente frente a las amenazas que impone el cambio climático.

La tercera contribución, realizada por Pamela Smith y Hugo Romero, cambia de escala y se adentra en la distribución de las temperaturas del aire al interior de los centros urbanos propiamente tal. Para tal efecto, se elabora un modelo de regresión entre la temperatura atmosférica, como variable dependiente y un conjunto de factores explicativos como cobertura vegetal, superficies impermeables, elevación topográfica, orientación de laderas, rugosidad, distancia a cursos de agua y admitancia térmica de coberturas de suelo en la metrópolis de Santiago. Los resultados muestran que el sector oriente de la ciudad presenta menores temperatruas que el resto de la ciudad y que los grandes parques presentan una diferencia de alrededor de $4^{\circ} \mathrm{C}$ más fría respecto a las zonas adyacentes.

A una escala más detallada, el trabajo Alexis E. Vásquez aborda el tema de la infraestructura verde y los servicios ecosistémicos del río Mapocho en Santiago y su área adyacente. Se evalúan tres servicios ecosistémicos claves del corredor ribereño: efecto enfriador, rutas para transporte no motorizado, y regulación de inundaciones, como elementos claves de mitigación y adaptación ante el cambio climático. Los resultados señalan que el servicio más importante de este corredor es la mitigación de emisiones de gases invernadero al servir como una importante ruta para el desplazamiento no motorizado, especialmente en bicicleta. La consolidación de una infraestructura verde asociada a este curso hídrico puede contribuir a mejorar la provisión de servicios ecosistémicos y de esta forma transformarse en una importante medida de mitigación y adaptación al cambio climático en Santiago.

Finalmente, el último aporte se dirige a la planificación y a la reflexión teórica sobre la adaptación urbana. El artículo de Jonathan Barton y Felipe Irarrázabal nos recuerda que el cambio climático no es un fenómeno nuevo y que no debe ser aislado de la histo- ria. Se estrucutura en dos partes: la primera corresponde a un análisis postestructural del concepto de riesgo urbano, así como de los conceptos de planificación, gestión y adaptación. Mientras que la segunda revisa la inclusión de riesgos hidrometeorológicos en la planificación urbana en distintas ciudades de Chile: Antofagasta, Coquimbo-La Serena, Valparaíso, Santiago y Concepción. Se concluye que aún hay trabajo pendiente, en investigación, en política pública e intervenciones específicas.

Como es tradición en Revista de Geografía Norte Grande, en este número 63 se publican otros cinco artículos que abordan diversas temáticas de interés.

La investigación de Antonio Daher, se focaliza en el estudio de lo que denomina como la "paradoja patagónica", correspondientes a las regiones chilenas de Aysén y Magallanes, las cuales si bien son contiguas y presentan similitudes geográficas, evidencian dinámicas económicas muy opuestas. Se perfila claramente que en los últimos cuarenta años, existen similitudes y diferencias sustantivas en los procesos económicos y cambios en la estructura del producto interno bruto de ambas regiones. Tales cambios estructurales revelan tanto el fuerte impacto de las políticas públicas explícitamente no territoriales o formuladas a-espacialmente (macroeconómicas, de apertura, fomento exportador e inserción global) como la acusada presencia estatal en ambas regiones de frontera.

El estudio Paula Núñez y Silvana López avanza sobre los debates asociados a la construcción jerárquica, pluriescalar, abierta y relacional de las regiones internas de la provincia de Río Negro en Argentina, ejemplificando cómo el reconocimiento y la transformación espacial habilita, paradójicamente, la asunción del espacio no solo como neutro, sino como marco justificativo de una integración social desigual, que para mediados de la década de 1970, comienza a plantearse en términos de determinismo geográfico, pues las desigualdades se justifican en descripciones del paisaje rionegrino. 
Arturo Vallejos-Romero, Alex Boso y Hugo Marcelo Zunino, abordan en su texto la relevancia que posee la confianza en las relaciones entre los actores sociales e instituciones públicas y privadas en los conflictos socioambientales. A partir de dos casos chilenos, la Termoeléctrica Castilla e Hidroeléctrica Hidroaysén en Chile, concluyen que la confianza es una dimensión relevante a la hora de dar cuenta de los riesgos y peligros asociados a conflictos socioambientales y que las probabilidades de decepción son mayores si este tipo de dimensiones sociales no son tomadas en cuenta. Frente a esto, es necesario que los diseños de intervención socioambiental incorporen tales dimensiones en pos de una mejor gobernanza de una sociedad moderna que produce y reproduce problemas y conflictos de compleja solución.

El artículo de María Isabel López Meza, Ignacio Bisbal y Leonel Pérez Bustamente, se enmarca en una línea de trabajo que resalta el legado de la actividad minera histórica como un tipo particular de patrimonio industrial. Aplicando en el territorio minero de Lota, epicentro del desarrollo de la industria del carbón en Chile, una metodología que integra el análisis cartográfico con la repetición de fotografías como técnica de análisis comparativo del paisaje histórico y contemporáneo, realiza un redescubrimiento del paisaje cultural a partir de los vestigios que de este quedan en el paisaje actual, favoreciendo la construcción de una interfaz cualitativa comprensible para las comunidades locales.

El último artículo publicado en este número es de Ángel Soria-Jáuregui, el cual realiza un estudio geomorfológico de la cuenca de Miranda, sector bisagra entre la depresión del Ebro y su cuenca alta, en España. Su objetivo fue definir la evolución geomorfológica cuaternaria a partir de diversos tipos de depósitos (laderas y fondo de valle), permitiendo establecer un modelo evolutivo de este sector. Para lograr esto, se realizó una caracterización sedimentológica, morfoestratigráfica y cronológica de dichas formaciones aluviales y coluvionares. Las fechas absolutas obtenidas a partir de los sedimentos fluviales del río Ebro permitieron la caracterización de su comportamiento hidrológico cuaternario.

Finalmente, en la sección de reseñas de libros, Simón Pedro Izcara Palacios analiza la obra editada por Francisco EntrenaDurán, Food Production and Eating Habits From Around the World: A Multidisciplinary Approach, el cual reúne una selección de trabajos escritos por especialistas en diversas disciplinas que abordan el estudio de la producción de comida y la construcción de los hábitos alimentarios en el interconectado mundo actual.

El conjunto de artículos publicados permite apreciar que las problemáticas e interrogantes espaciales son amplias y diversas; sin embargo, los impactos, vulnerabilidades y desafíos del cambio climático constituyen un quehacer mayor para los países, y por esa razón constituye el contenido principal de este numero semitemático. Ahora cabe preguntarse ¿están los instrumentos de planificación territorial y la institucionalidad asumiendo realmente este desafío? Sin duda esta y otras interrogantes, deben seguir suscitando el interés de la comunidad científica y los tomadores de decisión. Esperamos que estas contribuciones motiven y propicien nuevos aportes en estas materias. 
